\title{
Retour du congrès EuroMedLab par les internes Barcelone, 19-23 mai 2019
}

\author{
Aurore Desmous \\ Emma Galofaro \\ Mohamed Chtourou \\ Olivier Grunewald \\ Jeunes biologistes récipiendaires \\ des bourses SFBC \\ (Société française de biologie clinique) \\ <vsapin@chu-clermontferrand.fr>

\section{Session « Nouvelles pratiques dans le domaine des erreurs innées du métabolisme»}

Aurore Desmous

\section{La médecine de précision et le dépistage néonatal (DNN)}

\section{P. Rinaldo}

Etats-Unis

Le professeur Rinaldo présente la solution CLIR (Collaborative laboratory integrative reports) qui a pour objectif de limiter les cas de faux positifs retrouvés au cours du DNN. Il s'agit d'une application web gratuite, développée au sein de la Mayo Clinic qui a débuté en septembre 2018, et est destinée aux laboratoires de DNN pratiquant les dosages par des techniques de spectrométrie de masse. Cette application permet d'évaluer les valeurs de référence et valeurs seuils conventionnelles, et de les remplacer, si nécessaire, de façon à améliorer la décision clinique. La première étape a consisté à mettre en ligne les valeurs des dosages obtenus par l'ensemble des participants. Tous les cas avec des dosages positifs, définis à partir des valeurs conventionnelles, ont été analysés par biologie moléculaire. Cette analyse a permis de différencier les vrais des faux positifs et ainsi de redéfinir les valeurs de référence. Ainsi, les valeurs de référence du C14 : 1, dont le dosage permet le dépistage du déficit en VLCAD, ont été revues. Dans la maladie de Pompe, classiquement dépistée par une diminution de l'activité de la GAA (alpha-glucosidase acide), l'analyse des données a montré que l'association du rapport créatine/créatinine à l'activité GAA permettait une diminution drastique des faux positifs.

\section{La métabolomique non ciblée dans le diagnostic des désordres métaboliques}

\section{J. Jans}

Hollande

Les applications de la technique de métabolomique non ciblée développée au laboratoire d'Utrecht sont présentées. Cette technique est basée sur l'analyse d'échantillons de sang total recueilli sur papier buvard, de plasma ou de LCR, par infusion directe par spectrométrie de masse, sans étape de séparation chromatographique. La première application est le diagnostic des EIM. L'analyse des données a consisté à comparer l'intensité des pics de patients atteints d'EIM et de patients contrôles. Les patients atteints d'EIM ont été mis en évidence dans $88 \%$ des cas par cette approche. Les cas non mis en évidence étaient liés à des augmentations ou diminutions très faibles des métabolites, correspondant à des formes atténuées des maladies. Une deuxième application est la découverte de biomarqueurs en faisant une étude de métabolomique non ciblée chez 4 patients atteints d'une anomalie de la déglycosylation présentant des anomalies génétiques caractéristiques. Une autre application est la possibilité de mettre en évidence des anomalies de métabolites chez des patients n'ayant pas de diagnostic établi. Les patients, regroupés en fonction de leur atteinte clinique neurologique, et comparés à des patients contrôles, présentaient une augmentation d'un métabolite ayant la masse de l'IAA-RP (imidazole4-acetic acid-ribotide) dont l'impact a pu être démontré chez l'animal.

Les différents cas présentés montrent la nécessité de relier les anomalies phénotypiques aux anomalies génétiques, l'association de la métabolomique et de la génomique étant désignée sous le terme de cross-omics. 


\section{Diagnostic des EIM et NGS : opportunités et difficultés}

\author{
B. Pérez
}

Espagne

Le DNN comprend trois éléments : la stratification du patient, les études génétiques, qui sont détaillées dans cet exposé, et la génomique fonctionnelle. Pour les études génétiques, il existe quatre types d'approches : les panels de gènes (targeted exome sequencing), le séquençage de l'exome clinique (clinical exome sequencing), le séquençage de l'ensemble de l'exome (whole exome sequencing, WES), le séquençage de l'ensemble du génome (whole genome sequencing, WGS). Le choix de la technique se fera en fonction de la connaissance de la pathologie, de son hétérogénéité génétique et de la spécificité du phénotype. Ainsi, les panels de gènes sont adaptés lorsque le nombre de variants est faible et présentent comme limitation l'étude des séquences connues des gènes. Le DNN en Espagne est organisé en trois étapes : des premiers tests basés sur des techniques de SM avec l'analyse d'un nombre limité de métabolites, puis des tests de deuxième ligne utilisant différentes techniques et permettant d'analyser un plus grand nombre de métabolites (les acides aminés par LC-MS/MS, les acides organiques par GC-MS, les acylcarnitines par MS/MS), et enfin les tests génétiques permettant la confirmation des cas détectés. L'étude présentée évalue la possibilité de passer directement de l'étape 1 à la 3 , en s'affranchissant des analyses multiples par différents analyseurs. Les tests génétiques, réalisés par l'approche d'un panel de gènes, ont permis la confirmation des cas dans $40 \%$ (pour les anomalies de la bêta-oxydation des acides gras) à $80 \%$ des cas. Même si les techniques de génétique se sont beaucoup développées, la combinaison de la biochimie, de la génomique et de l'analyse fonctionnelle reste essentielle au diagnostic des EIM.

\section{Approches "intégratives » dans le diagnostic des EIM}

\section{H. Rocha}

Portugal

Les pratiques du diagnostic des EIM ont largement évolué depuis 1910, où la suspicion clinique constituait le point de départ et déclenchait la réalisation des analyses métaboliques. À partir des années 1960-70, dans le dépistage néonatal, les désordres des métabolites précèdent souvent les signes cliniques, et l'analyse génétique arrive en dernière ligne pour pouvoir valider les anomalies retrouvées. Dans certaines situations, où les prélèvements sont réalisés en période post mortem et où les marqueurs biochimiques ne sont pas forcément interprétables, le NGS peut avoir un intérêt en première ligne. Un cas de diagnostic de MADD (déficit multiple en acyl-CoA déshydrogénase) a été présenté et confirmé par NGS, le profil des acylcarnitines n'étant pas exploitable. Il s'agit d'un cas relativement rare puisque l'utilisation des analyses génétiques en première ligne peut générer des données délicates à interpréter, du fait de l'existence de nombreux variants de signification inconnue (vus, variants of uncertain significance). L'analyse des métabolites reste cruciale car elle permet de renseigner sur l'état métabolique du patient et d'orienter vers des investigations futures.

\section{Conférence "New generation biomarkers in infections"}

Emma Galofaro

\section{Conférence d'Emanuela Galliera}

PhD, Associate Professor, Dipartimento di Scienze Biomediche per la Salute, Universita degli Studi, Milano, IRCCS Galeazzi Orthopaedic Institute, Milano

L'infection des articulations prothétiques est la cause la plus fréquente des échecs d'arthroplastie. L'incidence de ces infections dépend de nature de l'articulation : de 1,7\% à 3,2 \% dans les prothèses de hanche ; et de $2,5 \%$ jusqu'à $5,6 \%$ pour les prothèses de genou. Le diagnostic précoce permet l'introduction rapide des antibiotiques et évite ainsi le plus possible les reprises de chirurgie. Ce diagnostic reste pourtant difficile du fait de la variabilité de la présentation clinique et de l'absence de gold standard diagnostic. Il est actuellement basé sur la clinique, les examens microbiologiques, l'histopathologie, l'imagerie et les marqueurs sanguins de l'inflammation.

Parmi ceux-ci, on trouve la CRP et la PCT. La première est peu informative dans ce contexte compte tenu d'une faible élévation, inférieure au seuil de $10 \mathrm{mg} / \mathrm{dL}$ dans les infections d'arthroplastie. La PCT, elle, est spécifique des infections systémiques bactériennes, mais ne présente pas d'augmentation très significative et n'apporte donc pas de valeur ajoutée pour ce type d'infection.

Le but de cette conférence était donc de présenter la pertinence d'autres marqueurs de l'inflammation en évaluant la significativité de l'augmentation de leurs concentrations sanguines dans les infections d'arthroplastie versus chez des patients non infectés.

Le marqueur uPAR (urokinase plasminogen activation receptor) permet la migration et l'adhésion des leucocytes dans les infections. Sa partie soluble (SuPAR) peut être retrouvée dans le sang et dans d'autres fluides organiques. 
Son augmentation est significative dans les infections d'arthroplastie (augmentation corrélée à celle des cytokines) et une concentration sanguine élevée de ce marqueur est prédictif de la sévérité de l'infection.

Parmi les toll-like receptors, TLR2 fait partie de la première ligne de défense contre les bactéries à Gram positif, tandis que TLR4 l'est pour les bactéries à Gram négatif. L'augmentation de la concentration sanguine de TLR2 est significative dans les infections d'arthroplastie tandis que celle de TLR4 ne l'est pas. Ceci peut être expliqué par le fait que les bactéries à Gram négatif ne représentent que $12,5 \%$ de ce type d'infection, et que la charge bactérienne pour déclencher l'infection est souvent plus faible que pour les bactéries à Gram positif. TLR2 peut donc être utilisé comme marqueur diagnostique, contrairement à TLR4.

Un autre marqueur pertinent est la présepsine, fragment soluble du CD14 (marqueur des monocytes). Son élévation, causée par la phagocytose bactérienne, est significative dans ce type d'infection. L'aire sous la courbe ROC de la présepsine est de 0,926, tandis que celles de la CRP et de l'IL-6 sont respectivement de 0,750 et 0,821 . Elle présente donc un intérêt pour le diagnostic. Elle a également une valeur pronostique grâce à une décroissance significative de sa concentration dès la $48^{\mathrm{e}}$ heure après chirurgie. Une faible diminution de sa concentration permet l'introduction rapide d'antibiotiques afin d'éviter une révision chirurgicale.

Selon les études de l'auteur, d'autres marqueurs permettent d'évaluer le pronostic du patient : SuPAR (AUC : 0,866), sTLR2 (AUC : 0,898), CCL2 (AUC : 0,868) et l'ostéopontine (AUC : 0,859 ). Ils sont tous impliqués dans la réponse inflammatoire médiée par les monocytes et les macrophages. Par ailleurs, certains marqueurs n'ont pas démontré de capacité diagnostique ni pronostique pour les infections d'arthroplastie : le sCD163 (AUC : 0,776), le sTREM-1 (AUC 0,751) et le MMP-9 (AUC : 0,597).

L'utilisation combinée de ces marqueurs permet d'obtenir une sensibilité et une spécificité élevées pour le diagnostic et le pronostic des infections d'arthroplastie.

Parmi eux, la présepsine présente une très bonne robustesse. Son utilisation est variée car elle a aussi montré une valeur diagnostique dans les sepsis néonataux, les pneumonies, les infections du système nerveux central, les infections fongiques et les infections cardiovasculaires.

\section{Symposium «HbA1c en pratique clinique »}

\section{Mohamed Chtourou}

\section{Diagnostic du diabète en utilisant HbA1C}

L'hémoglobine A1c (HbA1C) résulte de la réaction de glycosylation non enzymatique irréversible de la valine en position $\mathrm{N}$ terminale de la chaine $\beta$ de l'HbA. Elle reflète l'équilibre glycémique pendant une période de 8 à 12 semaines et permet le diagnostic et le suivi thérapeutique du diabète type 2 . C'est un marqueur prédictif des complications micro-et macro-vasculaires chez les diabétiques. Sa valeur normale est $<5,6 \%$.

Le diabète type 2 est diagnostiqué si l'HbAlc est $\geq 6,5 \%$ ( $\geq 48 \mathrm{mmol} / \mathrm{mol}$ ) et doit être confirmé par un deuxième dosage en dehors des patients qui sont symptomatiques ou qui ont une glycémie à jeun $>11 \mathrm{mmol} / \mathrm{L}$.

Pour une HbA1c entre 6 et $6,5 \%$ ou entre 5,6 et $6 \%$ associée à d'autres facteurs de risque de diabète, les patients sont à haut risque et doivent suivre des mesures préventives.

\section{Méthodes de dosages}

Le dosage de l'HbA1c se fait soit par méthodes séparatives selon la structure (chromatographie d'affinité) ou selon la charge (HPLC échangeuse d'ion, électrophorèse capillaire), soit par méthodes chimiques (immunologique ou enzymatique).

Il existe plusieurs sociétés savantes responsables du développement de ces différentes méthodes (NGSP pour USA, IFCC en Europe, autres pour la Suisse et le Japon). De ce fait l'IFCC a mis en place un processus de standardisation (standardisation des unités IFCC : $\mathrm{mmol} / \mathrm{mol}$ et NGSP (\%) en utilisant l'équation IFCC-NGSP).

\section{Limites de la HbA1c}

L'HbA1c ne peut pas être utilisée dans le diagnostic du diabète type 1 , lors de la présence d'un variant pathogène d'hémoglobine ( $\mathrm{HbS}, \mathrm{HbC} . .$.$) et la persistance héréditaire$ de l'hémoglobine foetale. Elle varie selon l'âge, l'ethnie.

Par ailleurs, les valeurs d'HbA1c sont affectées par l'altération de la fonction rénale et en cas d'anémie on peut observer des faux positifs.

\section{Standardisation de l'HbA1c}

Elle est basée sur la traçabilité métrologique qui permet la standardisation des tests aux niveaux des laboratoires médicaux (valeurs décisionnelles et recommandations). Un groupe de travail de l'IFCC a précisé la méthode de référence, les laboratoires de références et les critères d'acceptabilité de l'examen.

Ce groupe a développé un modèle pour classer les résultats des différents laboratoires, en fonction des techniques de dosage selon les critères d'acceptabilité (GOLD/SILVER/BRONZE/PASS/FAIL).

La limite d'acceptabilité est la ligne dessinée entre une imprécision de $5 \%$ pour IFCC (3,4\% NGSP) et un biais de $0,5 \mathrm{mmol} / \mathrm{mol}$ pour IFCC $(0,46 \%$ pour NGSP).

Le concept de EurA1c de l'IFCC a pour objectif principal de sensibiliser et d'améliorer les tests HbA1c en évaluant les performances des pays et des fabricants. Des échantillons de sang total frais et d'hémolysats lyophilisés synthétisés à 
partir du même pool ont été utilisés par 17 organisations du contrôle de qualité externe pour évaluer les performances analytiques de 2166 laboratoires. Les résultats ont été évalués par pays, par fabricant et par l'ensemble fabricant-pays selon les critères du modèle de l'IFCC. La majorité des résultats se trouvait dans la zone PASS et ils sont en train de s'améliorer. Tous les résultats ont été publiés en 2018 (doi:10.1373/clinchem.2018.288795).

Cette standardisation a une répercution sur le diagnostic et la classification des diabétiques (les valeurs nomales sont $<40 \mathrm{mmol} / \mathrm{mol}(5,8 \%)$, les valeurs pathologiques $>47 \mathrm{mmol} / \mathrm{mol}(6,4 \%)$, entre les deux il y a une zone dite grise dans laquelle les patients sont classés comme à haut risque. Donc, l'existence d'un bias ou d'une imprécision importante peuvent classer les patients différemment.

\section{POCT de I'HbA1c}

Une HbA1c élévée sans signes cliniques nécessite une confirmation par un autre prelèvement sanguin, ce qui peut retarder le diagnostic du diabète type 2 .

L'utilisation des instruments pour doser 1'HbA1c lors des consulations (point of care : POCT) vise à fournir des résultats rapides et de permettre une prise de décisions thérapeutiques dans les meilleurs délais, entraînant moins de visites et un meilleur contrôle de la glycémie.

Ces appareils doivent suivre les critères de la marque européenne CE ou American FAD, doivent être certifiés IFCC ou NGSP et doivent être évalués dans un laboratoire de biologie médicale, dans le site clinique et dans un laboratoire de référence.

Les critères décrits par NGSP pour ces appareils sont d'obtenir 36/40 échantillons au-dessous de $5 \%$ d'imprécision. L'erreur maximale acceptée par l'IFCC pour ces appareils est de $5 \mathrm{mmol} / \mathrm{mol}$ pour un taux d'HbA1c de $50 \mathrm{mmol} / \mathrm{mol}$.

En conclusion, les critères d'acceptabilité doivent être les mêmes, quelle que soit la méthode utilisée, dosage en POCT ou au laboratoire et quelque soit l'objectif clinique, diagnostic ou suivi thérapeutique.

\section{Vers la biologie et au-delà}

\section{Olivier Grunewald}

Interne en biologie médicale $8^{\mathrm{e}}$ semestre, Laboratoire de génétique, CHU de Lille, France

Cela fait maintenant bientôt quatre ans que j'ai débuté mon internat en biologie médicale. Spécialité choisie au terme des ECN (épreuves classantes nationales) parmi les trente proposées pour ses nombreux attraits : une discipline où la théorie et la pratique sont indissociables, où l'on apprend à maîtriser, choisir et améliorer ses techniques, le tout dans un champ d'action presque infini de thématiques médicales. Au cours de ces années d'internat, pendant lesquelles j'ai axé ma formation sur le diagnostic des maladies rares par séquençage à haut débit, j'ai pu explorer divers aspects de la biologie médicale en France (en rejoignant les associations et syndicats d'internes et en participant à divers congrès nationaux). Il me restait à pousser mes recherches au niveau européen. Comment pratique-t-on la biologie chez nos voisins ? Comment travaillent nos confrères ? Que pouvons-nous apprendre d'eux et ramener en France pour nous améliorer?

C'est ainsi que je me suis décidé à participer à l'EuroMedLab 2019, avec pour objectifs de découvrir la biologie médicale européenne, de rencontrer les membres de la Young scientist task force (IFCC YSTF) et d'assister aux présentations traitant des nouvelles technologies dans la recherche des maladies rares.

\section{Impressions générales sur le congrès}

Ce congrès était tout simplement impressionnant. Plus de 6000 participants sur 4 jours, provenant de plus de 80 pays (ce qui dépasse très largement les frontières de l'Europe) ; 1500 posters présentés, des dizaines de conférences, symposiums, ateliers (impossible d'assister à tous). L'alternance entre les conférences scientifiques et les ateliers sponsorisés permettait à chacun de se faire son programme et de satisfaire tous les participants. Quant à l'exposition, jamais je n'avais vu autant d'acteurs de l'industrie du diagnostic in vitro, trois jours n'ont pas suffi à en faire le tour.

J'ai également été agréablement surpris du grand nombre de Young delegates (ndlr comprendre moins de 35 ans), qui laisse présager que la biologie médicale est jeune, dynamique et a encore un bel avenir européen.

\section{Symposium : Principes et applications de l'épigénétique}

À l'heure où l'on est capable de séquencer l'intégralité du génome humain pour quelques centaines d'euros en une journée, on n' arrive toujours pas à identifier l'ensemble des maladies héréditaires. Viennent donc se greffer à la génomique, depuis quelques années, de nouvelles disciplines. L'épigénétique est l'une d'elles. Elle cherche à expliquer comment est régulée l'expression des gènes par des mécanismes biochimiques réversibles et transmissibles.

Les variations de la méthylation de l'ADN par exemple : en ajoutant un groupement méthyl aux bases cytosines des îlots « $\mathrm{CpG}$ », très représentés dans les régions promotrices régulatrices des gènes, on va pouvoir mettre ceux-ci sous silence, et diminuer leur expression.

Les histones : ces protéines nucléolaires qui ont pour but de condenser l'ADN. Des modifications post-traductionnelles 
de ces histones vont permettre, selon les besoins de la cellule, de condenser ou de libérer certaines régions génomiques, qui pourront alors être transcrites ou, au contraire, mises sous silence.

Ces différentes mécaniques, par ailleurs, interagissent entre elles et son déjà étudiées en pathologie humaine, dans les anomalies du développement, le vieillissement, les maladies métaboliques ou cardiovasculaires...

\section{Viewpoint : YSTF session}

Cette première session organisée par la YSTF nous a présenté différentes structures et associations de jeunes biologistes à travers l'Europe (l'association française étant la FNSIP-BM). Nous avions déjà rencontré l'AABC (association belge) via des programmes d'échanges locaux, nous pourrons je l'espère les étendre vers d'autres horizons.

Une présentation didactique, par la suite, nous a donné les « 10 astuces » pour publier ses travaux dans les meilleures revues scientifiques.

Nous avons ensuite eu des retours d'expériences de jeunes biologistes de la YSTF, sur leurs travaux de recherche, leurs mobilités internationales ou leurs carrières.

\section{Viewpoint : CRISPR}

Quiconque s'intéresse aux maladies héréditaires et à la génomique a déjà dû entendre parler du CRISPR (Clustered regular interspaced short palindromic repeats), en particulier du CRISPR-cas9 : les « ciseaux à ADN ». Cette technologie permet de couper les brins d'ADN à un endroit donné, ce qui permet d'inactiver un gène ou même d'introduire du matériel génétique. Cet outil est attendu par la communauté scientifique pour son intérêt majeur en thérapie génique. Cependant, plusieurs difficultés restent cependant à prendre en compte, d'une part, les problèmes inhérents à la technique (reconnaissance de cette enzyme bactérienne par le système immunitaire de l'hôte ou encore le phénomène de off-targets cutting qui reste le principal obstacle), d'autre part, les problèmes éthiques qu'une telle application peut poser. Cette technologie d'avenir devra donc encore être étudiée et perfectionnée avant de pouvoir être utilisée en thérapeutique humaine.

\section{Symposium : La formation à la médecine de laboratoire en Europe - en partenariat avec I'IFCC YSTF}

Cette seconde session de la YSTF traitait de la formation des jeunes biologistes. Quelles sont les opportunités qui nous sont offertes (en recherche comme en hospitalier) ? Quels sont les obstacles à franchir ? Comment pouvoir accéder à un programme de mobilité international ? Quelles sont les attentes des jeunes biologistes?

Selon les pays, cette formation peut être très différente (formation scientifique ou santé, activité de recherche ou pas, activité clinique ou pas...), c'est néanmoins cette diversité des profils qui contribue à la polyvalence de la discipline, à la frontière entre le soin et la recherche.

Plénière : DPNI et analyse de l'ADN libre circulant, transfert de l'innovation vers la pratique clinique

L'étude de l'ADN libre circulant a rapidement trouvé sa place dans le monde du diagnostic prénatal, si bien que les recommandations de dépistage de la trisomie 21 ont récemment été modifiées pour intégrer ce test, ce qui a pu diminuer fortement les examens invasifs dans plusieurs pays. Néanmoins les possibilités de cette technologie ne s'arrêtent pas là.

En effet, le Docteur Rossa CHIU (Université de Hong Kong) nous a présenté ses travaux permettant la recherche de maladies monogéniques par l'étude de l'ADN libre fœtal, notamment dans le cadre des maladies liées à l'X (en particulier pour les fotus masculins).

En dehors du diagnostic prénatal, l'ADN libre circulant peut également être utilisé en cancérologie, en détectant de multiples anomalies chromosomiques, on peut suspecter des réarrangements responsables d'hémopathies malignes. D'autres applications en dépistage pré-symptomatique de néoplasies ORL sont en cours d'études par cette équipe.

Ces fragments d'ADN circulant peuvent donc être étudiés de nombreuses façons : séquençage, quantification, longueur des fragments, points de cassures... Toutes ces informations combinées forment ainsi la " fragmentomique », une technologie qui va sans aucun doute se développer dans les années à venir.

\section{Symposium : Les mégadonnées (big data) en biologie médicale}

Génomique, transcriptomique, protéomique, épigénétique, métabolomique, fragmentomique... toutes ces nouvelles technologies génèrent des quantités hallucinantes de données de santé, données à ajouter aux données cliniques, à l'imagerie, aux traitements et aux résultats biologiques du patient. Le traitement, l'utilisation, le stockage et la sécurisation de ces mégadonnées sera bientôt un enjeu majeur pour les laboratoires, d'autant plus que le dossier médical partagé n'a jamais été aussi proche d'aboutir. Faut-il dès aujourd'hui former les laboratoires à la bioinformatique ou bien ceux-ci feront-ils appels à des entreprises extérieures ? Maîtriser son examen de laboratoire c'est également maîtriser le travail des données, il n'est pas concevable d'avoir une «boîte noire » informatique sans avoir un minimum de connaissances du biologiste à ce sujet.

Les objets connectés sont également devenus des accessoires du quotidien des Français, et parmi eux certains génèrent des données de santé (fréquence cardiaque, tension, étude biochimique de la sueur...). Le recueil et 


\section{Congrès}

l'utilisation des données biologiques par les biologistes seront également nécessaires pour ne pas tomber dans le mésusage de ces informations.

\section{Autres aspects d'EuroMedLab}

En dehors des conférences, de l'exposition des partenaires et des posters, ce congrès m'a également permis de rencontrer d'autres jeunes biologistes. J'y ai d'abord revu des connaissances d'autres universités de France ainsi que nos voisins belges, que nous avions déjà vus à plusieurs reprises, mais l'EuroMedLab a été l'occasion de rencontrer des biologistes italiens, espagnols, polonais, serbes et même indiens. J'espère pouvoir entretenir et développer ces relations grâce à l'EFLMLabX ou la plateforme Lab Surfing.

Cela a également été l'occasion pour moi de présenter mon travail de thèse lors de la session poster aux quelques curieux intéressés par le diagnostic moléculaire des néphropathies héréditaires.

\section{Conclusion}

La biologie médicale en France traverse en ce moment une période de doute. Son attractivité auprès des jeunes a dégringolé ces dernières années, les contraintes liées à l'accréditation et aux regroupements des laboratoires empiètent sur le temps médical des biologistes, qui sont de plus en plus isolés dans leurs laboratoires. Cependant, les innovations technologiques se multiplient, repoussant toujours plus loin les limites de notre compréhension du corps humain. Les nouveaux automates sont de plus en plus précis, de plus en plus rapides, ce qui nous permettra de libérer du temps pour renouer le dialogue avec nos confrères cliniciens et avec les malades. La biologie délocalisée, la santé connectée, le partage des données, les technologies « omiques » sont autant de défis à relever pour les biologistes, mais rien qu'à voir les milliers de participants qui sont venus à l'EuroMedLab 2019 à Barcelone, j'entrevois un bel avenir à mon futur métier.

Rendez-vous pour l'\#IFCCWorldLab2020 à Séoul.

Remerciements. Je remercie la Société française de biologie clinique de permettre aux jeunes biologistes de participer à ces évènements grâce à leur programme de financement de congrès, sans lequel je n'aurai pas pu assister à l'EuroMedLab 2019. Je remercie également les autres membres de notre « délégation lilloise », Jean-David, Guillaume et Benjamin de m'avoir accompagné lors de ce séjour catalan. 